\title{
Ecologia alimentar da corvina, Pachyurus bonariensis (Perciformes, Sciaenidae) em duas baías do Pantanal, Mato Grosso, Brasil
}

\author{
Rosemara Fugi, Norma S. Hahn, Gisele C. Novakowski \& Graziela C. Balassa
}

Núcleo de Pesquisas em Limnologia, Ictiologia e Aqüicultura (Nupélia) da Universidade Estadual de Maringá, Av. Colombo 5790, 9020900 Maringá, Paraná, Brasil. (rfugi@nupelia.uem.br).

\begin{abstract}
Feeding ecology of Pachyurus bonariensis (Perciformes, Sciaenidae) in two bays of the Pantanal, Mato Grosso State, Brazil. Spatial, temporal and ontogenetic variations in the diet of Pachyurus bonariensis Steindachner, 1879 were analyzed. Fishes were taken monthly from March 2000 to February 2001 and from August 2002 to February 2003 in the Chacororé and Sinhá Mariana bays. Diet was determined analyzing 359 stomach contents. To express diet results, were used occurrence and volumetric methods. The individuals consumed expressive amount of Chironomidae and Ephemeroptera larvae, in both bays and periods (dry and wet). Ontogenetic analysis showed that small individuals (Standard length $=1.6$ to $7.2 \mathrm{~cm}$ ) ate mainly Chironomidae, whereas larger ones (Standard length $=13.0$ to $18.6 \mathrm{~cm}$ ) consumed mainly Ephemeroptera. Then it is possible to infer that P. bonariensis is benthic-feeding fish with specialized diet. This characteristic was attributed because its narrow niche breadth allied with position and form of its mouth adapted to feed on the bottom.
\end{abstract}

KEYWORDS. Feeding, fishes, bays, Pantanal.

RESUMO. Foi investigada a dieta de Pachyurus bonariensis Steindachner, 1879 associando-a à variações espaciais, temporais e ontogenéticas. Os peixes foram coletados mensalmente de março/2000 a fevereiro/2001 e de agosto/2002 a fevereiro/2003 em duas baías do Pantanal, Chacororé e Sinhá Mariana. Conteúdos estomacais de 359 exemplares foram analisados através dos métodos de freqüência de ocorrência e volumétrico. A dieta foi composta essencialmente por larvas bentônicas de Chironomidae e Ephemeroptera, independente do local e período (seca e cheia) analisados. Entretanto, nota-se marcante diferença na composição alimentar de acordo com o tamanho do peixe. Os menores exemplares (comprimento padrão $=1,6$ a 7,2 $\mathrm{cm}$ ) consumiram preferencialmente Chironomidae e os maiores (comprimento padrão $=13,0$ a 18,6 cm) Ephemeroptera. Conclui-se que $P$. bonariensis tem hábito alimentar bentívoro e especializado, devido principalmente ao restrito espectro alimentar aliado à posição e forma da boca adaptada a explorar o substrato.

PALAVRAS-CHAVE. Alimentação, peixes, baías, Pantanal.

O estudo de hábito alimentar em peixes, mesmo que em caráter descritivo, fornece informações fundamentais sobre a autoecologia de uma espécie. A despeito da complexidade apresentada pelos ecossistemas de água doce no que diz respeito às relações tróficas, o conhecimento da alimentação natural baseado na análise do conteúdo estomacal tem sido utilizado como base para o entendimento do papel ecológico desempenhado pelas espécies (WINDELL \& BowEN, 1978).

Uma questão fundamental na ecologia trófica é identificar os fatores que determinam o modelo de utilização do alimento por peixes (WAINWRIGHT, 1988), pois vários deles podem estar envolvidos no processo de forrageamento. Durante o ciclo de vida, muitos peixes alteram a dieta, principalmente em função do crescimento (HAHN et al., 2000; ORTÊNCIO-FILHO et al., 2001; LIMAJunior \& GoITEN, 2003) e da disponibilidade de recursos alimentares (Lowe-McConnel, 1987; AвELHA et al., 2001) permitindo, que indivíduos de uma mesma espécie apresentem diferenças sazonais (HAHN et al., 1997) e/ou espaciais (Agostinho et al., 1997; AbujanRa et al., 1999) relacionadas à alimentação.

A corvina de rio, Pachyurus bonariensis Steindachner, 1879 foi uma das espécies mais capturadas nas baías Chacororé e Sinhá Mariana durante a pesca experimental do projeto "Biologia pesqueira e pesca na área de influência do reservatório de Manso: biologia e socioeconomia". Nestas baías coexistem aproximadamente 157 espécies de peixes (Fugi, dados não publicados), cujos registros biológicos e/ou ecológicos, são escassos ou inexistentes na literatura, para a maioria delas. $\mathrm{Na}$ água doce são encontrados seis gêneros da família Sciaenidae, dos quais Pachyurus, Plagioscion, Pachypops e Petilipinnis são endêmicos da América do Sul (CASATTI, 2003). Trata-se de uma espécie bentopelágica de pequeno porte, que não ultrapassa $22,6 \mathrm{~cm}$ de comprimento padrão (CAsatTi, 2003).

Considerando a elevada diversidade ictiofaunística na região estudada, o papel biológico que uma espécie dominante pode exercer sobre as outras e o entendimento das relações tróficas, este estudo buscou elucidar aspectos da ecologia trófica de $P$. bonariensis com ênfase nas escalas espacial, temporal e ontogenética, de modo a contribuir para a preservação da ictiofauna.

\section{MATERIAL E MÉTODOS}

Os peixes foram coletados nas baías Chacororé e Sinhá Mariana, localizadas no complexo de baías e várzeas do Pantanal no município de Barão de Melgaço (MT), $300 \mathrm{~km}$ a jusante do reservatório de Manso. Situadas na margem esquerda do rio Cuiabá (Fig. 1), estas duas baías estão constantemente conectadas, perdendo a comunicação apenas em condições de seca extrema.

As coletas foram realizadas mensalmente de março/ 2000 a fevereiro/2001 e de agosto/2002 a fevereiro/2003. Para as capturas foram utilizadas 14 redes de espera (10 $\mathrm{m}$ de comprimento cada uma) com diferentes tamanhos 
de malhas $(2 ; 4 ; 3 ; 4 ; 5 ; 6 ; 7 ; 8 ; 9 ; 10 ; 12 ; 14 ; 16 ; 18$ e $30 \mathrm{~cm}$ entre nós opostos) expostas por 24 horas, com revistas às 8:00, 16:00 e 22:00 horas. Nas áreas litorâneas das baías foram operadas redes de arrasto (20 $\mathrm{m}$ de comprimento e malha de $1 \mathrm{~cm}$ entre nós opostos) durante o dia e à noite. Todos os exemplares foram identificados, medidos e pesados e seus estômagos foram retirados e fixados em formol $4 \%$. Exemplares-testemunho desta espécie foram depositados na Coleção Ictiológica do Núcleo de Pesquisas em Limnologia, Ictiologia e Aqüicultura (Nupélia) sob número NUP 988.

Os conteúdos estomacais foram analisados através dos métodos de frequiência de ocorrência e volumétrico (Hyslop, 1980). O volume dos itens alimentares foi obtido por deslocamento da coluna de água, utilizando-se uma bateria de provetas graduadas. Para itens menores que $0,1 \mathrm{ml}$, foi utilizada uma placa milimetrada onde o volume foi obtido em $\mathrm{mm}^{3} \mathrm{e}$ posteriormente transformado em $\mathrm{ml}$ (Hellawell \& Abel, 1971).

A dieta foi avaliada por local (baías Chacororé e Sinhá Mariana), por período do ano (seca e cheia) e por classes de tamanho. O período de seca correspondeu aos meses de maio, junho, julho, agosto, setembro e outubro e os de cheia aos meses de novembro, dezembro, janeiro, fevereiro, março e abril, independente do ano de coleta. Para a análise por classes de tamanho (avaliada apenas pelo método volumétrico) os exemplares foram separados em três grupos de acordo com a variação do comprimento padrão: 1,6 a 7,2 cm; 7,3 a 12,9 cm; e de 13,0 a $18,6 \mathrm{~cm}$. Todos os indivíduos na última classe de tamanho eram adultos.

\section{RESULTADOS}

A análise por local, período e classes de tamanho de 359 exemplares de $P$. bonariensis, cujo comprimento padrão variou de 1,6 a 18,6 cm revelou uma dieta composta por Chironomidae, Ephemeroptera, Diptera (Chaoboridae e Ceratopogonidae), outros insetos (Coleoptera, Trichoptera, Hemiptera e restos não identificados), microcrustáceos (Ostracoda, Cladocera, Copepoda e Conchostraca) e vegetais. A dieta baseou-se predominantemente em insetos aquáticos, sendo que dentre estes se destacaram Chironomidae e Ephemeroptera, tanto na baía Chacororé quanto na Sinhá Mariana (Tab. I).

$\mathrm{Na}$ baía Chacororé durante o período de seca, Chironomidade e Ephemeroptera foram os únicos itens consumidos pela espécie. Neste período, Chironomidae foi o alimento mais importante tanto em freqüência de ocorrência $(86,4 \%)$ quanto em volume $(79,6 \%)$, enquanto Ephemeroptera foi consumido por poucos exemplares $(20,4 \%)$ e com volume menos representativo $(18,2 \%)$ (Fig. 2). No período de cheia, embora Ephemeroptera e Chironomidae tenham sido os itens mais consumidos, verificou-se que a dieta foi mais diversificada, sendo registrados outros insetos e matéria vegetal. Ao contrário do verificado na seca, Ephemeroptera foi predominante na cheia, tendo ocorrido em 69,9\% dos estômagos analisados (84,6\% do volume consumido) (Fig. 2). Embora Chironomidae tenha representado uma pequena parcela em volume $(11,7 \%)$, ocorreu em $75,9 \%$ dos conteúdos estomacais dos exemplares analisados na cheia (Fig. 2).
Na baía Sinhá Mariana (Fig. 3), no período de seca, Chironomidae e Ephemeroptera também foram os únicos itens consumidos. Chironomidae foi um recurso explorado por muitos exemplares $(94,7 \%$ em freqüência de ocorrência), entretanto, sua participação em volume foi menos expressiva $(31,1 \%)$. Por outro lado, Ephemeroptera obteve um volume expressivo $(68,9 \%)$ mas foi consumido por poucos exemplares $(19,3 \%$ em freqüência de ocorrência) (Fig. 3). No período de cheia constatou-se também uma maior variedade de itens na dieta. Chironomidae foi o alimento principal, tanto em ocorrência $(94,9 \%)$ quanto em volume $(56,2 \%)$, enquanto Ephemeroptera teve participação importante em volume (40\%), porém pequena em ocorrência $(14,2 \%)$ (Fig. 3). Os demais itens registrados apresentaram valores inexpressivos, com exceção de Diptera e microcrustáceos, importantes apenas em ocorrência $(22,3$ e $13,2 \%$, respectivamente) (Fig. 3).

A análise da dieta por classes de tamanho revelou para ambas as baías um padrão consistente (Fig. 4). Os menores exemplares analisados $(1,6 \mathrm{~cm} \mathrm{a} 7,2 \mathrm{~cm})$ consumiram preferencialmente Chironomidae $(78,6 \mathrm{e}$

Tabela I. Número de estômagos analisados de exemplares de Pachyurus bonariensis Steindachner, 1879 por local de coleta (baías Chacororé e Sinhá Mariana, Mato Grosso), período do ano (seca e cheia) e classes de tamanho, coletados de março/2000 a fevereiro/2001 e de agosto/2002 a fevereiro/2003.

\begin{tabular}{lcrcrr}
\hline $\begin{array}{l}\text { Classes de } \\
\text { tamanho }(\mathrm{cm})\end{array}$ & \multicolumn{2}{c}{$\begin{array}{c}\text { Chacororé } \\
\text { seca }\end{array}$} & \multicolumn{2}{c}{ chinha Mariana } & Total \\
\hline $1,6-7,2$ & 19 & 13 & 44 & 163 & 239 \\
$7,3-12,9$ & 2 & 7 & 12 & 18 & 39 \\
$13,0-18,6$ & 1 & 63 & 1 & 16 & 81 \\
Total & 22 & 83 & 57 & 197 & 359 \\
\hline
\end{tabular}
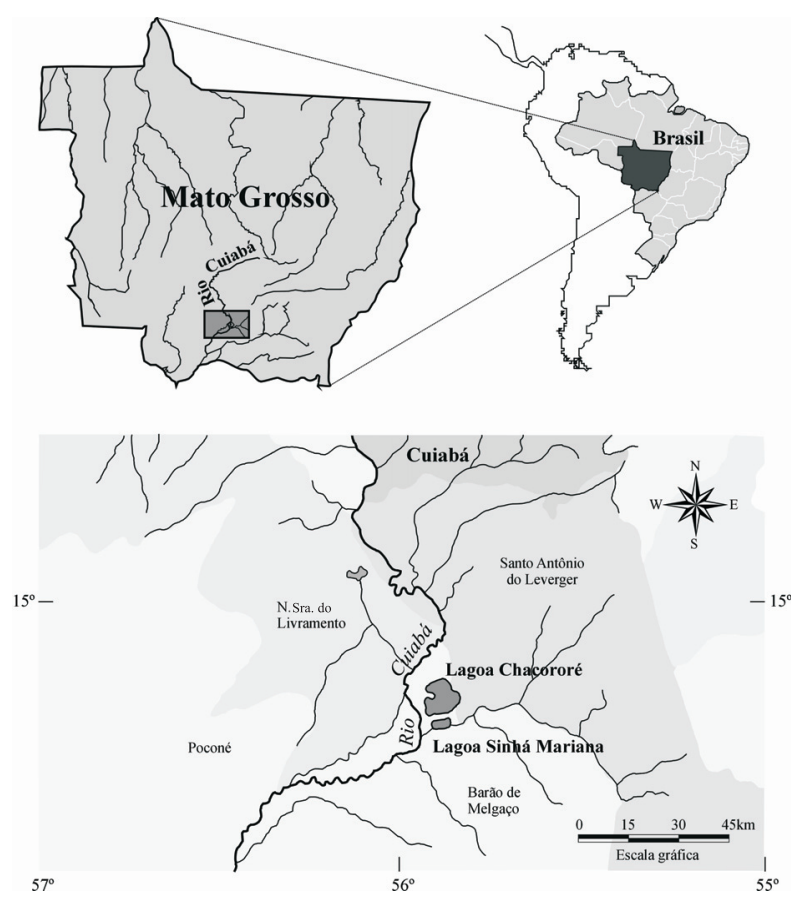

Fig. 1. Localização das estações de amostragem: baías Chacororé e Sinhá Mariana, Mato Grosso. 

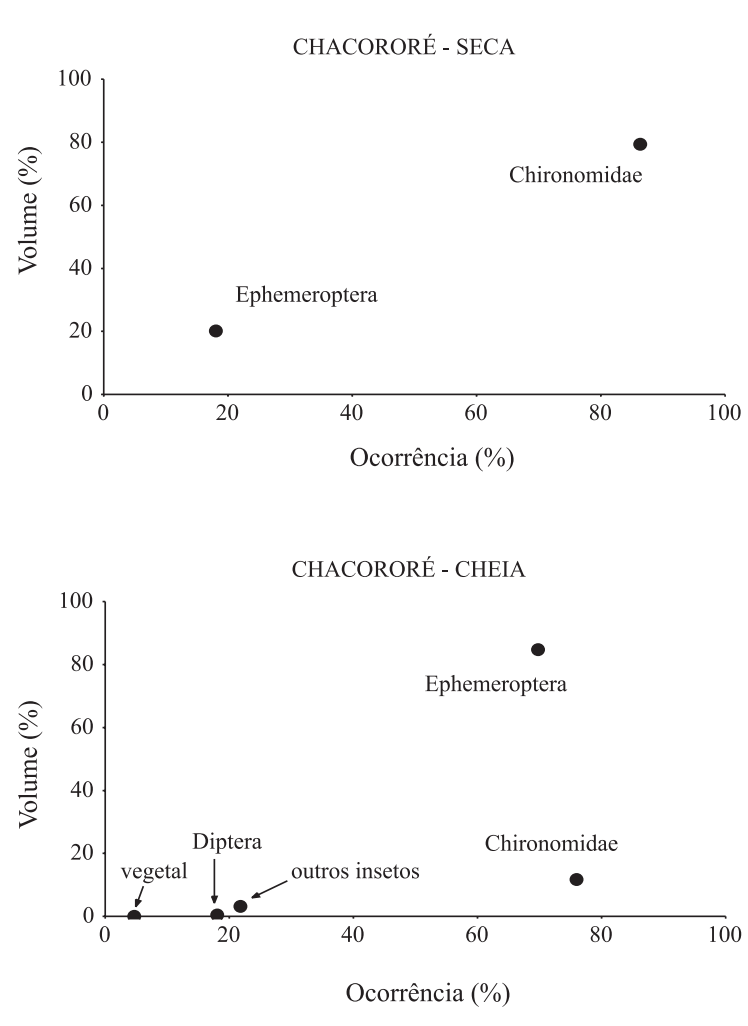

Fig. 2. Variações sazonais (seca e cheia) na composição da dieta de Pachyurus bonariensis Steindachner, 1879 na baía Chacororé, Mato Grosso, de março/2000 a fevereiro/2001 e de agosto/2002 a fevereiro/2003.
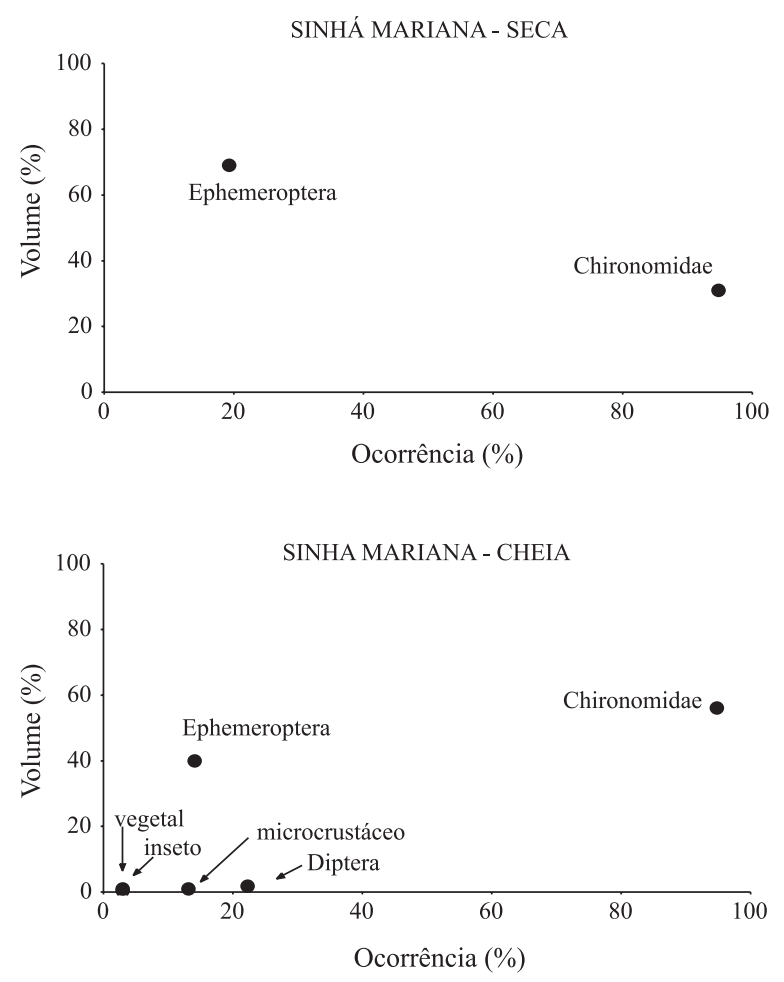

Fig. 3. Variações sazonais (seca e cheia) na composição da dieta de Pachyurus bonariensis Steindachner, 1879 na baia Sinhá Mariana, Mato Grosso, de março/2000 a fevereiro/2001 e de agosto/2002 a fevereiro/2003.
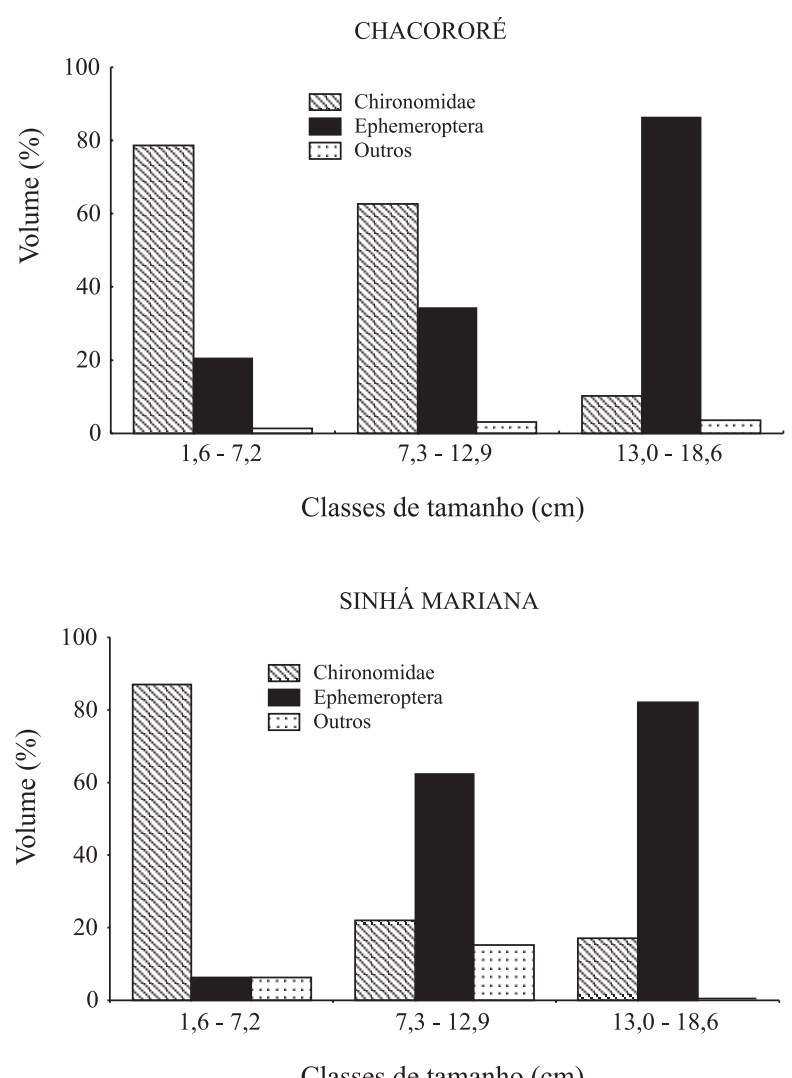

Fig. 4. Composição da dieta de Pachyurus bonariensis Steindachner, 1879 por classes de tamanho nas baías Chacororé e Sinhá Mariana, Mato Grosso, de março/2000 a fevereiro/2001 e de agosto/2002 a fevereiro/2003.

87,1\% na Chacororé e Sinhá Mariana, respectivamente) (Fig. 4). Os exemplares de tamanho intermediário $(7,3 \mathrm{~cm}$ a $12,9 \mathrm{~cm}$ ) alimentaram-se principalmente de Chironomidade (62,6\%), seguido de Ephemeroptera $(34,5 \%)$ na Chacororé e de Ephemeroptera $(62,4 \%)$ seguido de Chironomidae (22,2\%) na Sinhá Mariana (Fig. 4). Os maiores exemplares $(13,0 \mathrm{~cm}$ a $18,6 \mathrm{~cm})$ exploraram basicamente Ephemeroptera, tanto na Chacororé $(86,3 \%)$ como na Sinhá Mariana (82,2\%) (Fig. 4).

\section{DISCUSSÃO}

Pachyurus bonariensis explorou poucas fontes de alimento e com base nas características das presas (quase que exclusivamente larvas bentônicas de insetos) e na morfologia da boca (pequena e em posição sub-inferior) (Fugi, dados não publicados) sugere-se que esse peixe forrageia no substrato, o que caracteriza um hábito bentívoro. No rio São Francisco, Pachyurus squamipinnis (Agassiz, 1829) e Pachyurus francisci (Cuvier, 1830) foram caracterizadas como piscívoras (ALVIM \& PERET, 2004), hábito atribuído também a outro Sciaenidae, Plagioscion squamosissimus (Heckel, 1840) na planície de inundação do alto rio Paraná (HaHn et al., 1997; AlmEIDA et al., 1997). No entanto, na área de influência da represa Capivara, $P$. squamosissimus consumiu grandes quantidades de camarões, além de peixes (BENNEMANn et al., 2006). 
Chironomidae e Ephemeroptera foram presas dominantes nos conteúdos estomacais analisados, independente do local e época do ano em que os exemplares foram coletados. Destaca-se, no entanto, que embora ambos tenham sido dominantes na dieta, Chironomidae foi mais importante em ocorrência e Ephemeroptera em volume ingerido (evidenciando o maior tamanho deste último grupo de insetos). Esses mesmos recursos se destacaram nos conteúdos estomacais de Pachyurus squamipinnis, capturados no reservatório de Três Marias, MG, embora os maiores exemplares $(\mathrm{CP}=30 \mathrm{a} 40 \mathrm{~cm})$, tenham consumido peixes (Mourão \& Torres, 1984). Ambos os grupos de insetos são muito comuns em águas continentais, onde passam uma grande parte de seu desenvolvimento (TAKEDA \& Fusita, 2004). Para os peixes, eles representam uma importante fonte de energia e são muito freqüentes nos conteúdos estomacais de espécies que exploram o substrato como Iheringichthys labrosus (Lütken, 1874), Trachydoras paraguayensis (Eigenmann \& Ward, 1907) e Leporinus amblyrhynchus Garavello \& Britski, 1987 (Fugi et al., 1996; DuRÃEs et al., 2001; MENDONÇA et al., 2004). Diversos autores comentam sobre a importância das larvas de insetos na alimentação dos peixes, considerando que são elementos constantes na dieta de diversas espécies de água doce (SABINO \& CASTRO, 1990; Luiz et al., 1998; Russo et al., 2002). Segundo GaLina \& HAHN (2004), a elevada quantidade de larvas de Chironomidae encontrada nos conteúdos gástricos de peixes pode refletir a ampla distribuição desses insetos, bem como a adaptabilidade a praticamente qualquer tipo de ambiente aquático.

A maior diversidade de itens na dieta da espécie durante o período de águas altas pode ser um reflexo da ampliação do ambiente, o qual incorpora novos microhábitats conforme vai sendo inundado, trazendo novos recursos alimentares para os peixes. Para YАмАмото (2004), a oferta de alimentos é elevada na enchente e cheia, ampliando o espectro alimentar de muitos peixes, que se torna restrito na seca. De acordo com Prejs \& Prejs (1987), em rios onde ocorrem inundações, os ciclos são de abundância de recursos na estação chuvosa e limitação na estação seca. Assim, o consumo exclusivo de Chironomidae e Ephemeroptera na seca sugere que, em períodos de menor disponibilidade de recursos, $P$. bonariensis restringe seu espectro alimentar tornando-se mais especialista. Este padrão foi mencionado por ZARET \& RAND (1971) para algumas espécies de peixes em um riacho tropical.

O elevado consumo de Chironomidae e Ephemeroptera nos dois locais amostrados tanto na seca quanto na cheia indicam que $P$. bonariensis apresenta preferência por estes invertebrados, e o fato de não ter sido registrada matéria inorgânica nos conteúdos estomacais sugere que ocorre seleção do alimento na cavidade bucal. LAMMENS \& HoogenBoEZEM (1991) observaram que peixes bentívoros eliminaram pequenas partículas de areia através dos rastros branquiais. Este mecanismo foi atribuído a $I$. labrosus que consome elevada quantidade de larvas de Chironomidae e tem rastros branquiais bem espaçados permitindo a retenção das larvas e a rejeição de matéria inorgânica (Fugr et al., 2001). Comportamento semelhante foi descrito para o Sciaenidae marinho Umbrina coroides (Cuvier, 1830), espécie bentívora que se alimenta de crustáceos e elimina areia através da boca e rastros branquiais (ZAHORCSAK, et al., 2000).

As variações na dieta por classes de tamanho, que mostraram uma alteração gradativa no consumo de Chironomidae e Ephemeroptera é um forte indício de seleção do predador pelo tamanho de presa. Este padrão de variação ontogenética na dieta tem sido descrito para várias espécies de peixes de água doce (HAHN et al., 2000; ORTÊNCIO-FILHO et al., 2001; LiMA-JunIOR \& GOITEN, 2003; NovaKowsKi et al., 2004). Desse modo, sugere-se que as diferenças encontradas na dieta de $P$. bonariensis são determinadas principalmente pelo tamanho dos peixes, uma vez que em ambas as baías, tanto na seca quanto na cheia, Chironomidae e Ephemeroptera foram os alimentos dominantes na dieta.

Agradecimentos. Os autores agradecem ao Convênio UEM/Nupélia/Furnas Centrais Elétricas pelo apoio financeiro e ao Núcleo de Pesquisas em Limnologia, Ictiologia e Aqüicultura (Nupélia) pela infraestrutura concedida.

\section{REFERÊNCIAS BIBLIOGRÁFICAS}

Abelha, M. C. F.; Agostinho, A. A. \& Goulart, E. 2001. Plasticidade trófica em peixes de água doce. Acta Scientiarum 23(2): 425-434.

Abujanra, F.; Russo M. R. \& Hahn, N. S. 1999. Variações espaçotemporais na alimentação de Pimelodus ortmanni (Siluriformes, Pimelodidae) no reservatório de Segredo e áreas adjacentes (PR). Acta Scientiarum 21(2):283-289.

Agostinho, A. A.; Ferreti, C. M. L.; Gomes, L. C.; Hahn, N. S.; Suzuki, H. I.; Fugi, R. \& Abujanra, F. 1997. Ictiofauna de dois reservatórios do rio Iguaçu em diferentes fases de colonização: Segredo e Foz do Areia. In: Agostinho, A. A. \& Gomes, L. C. eds. Reservatório de Segredo: bases ecológicas para o manejo. Maringá, EDUEM. p.275-292.

Almeida, V. L. L.; Hahn, N. S. \& Vazzoler, A. E. A. M., 1997. Feeding patterns in five predatory fishes of the high Paraná River floodplain (PR, Brazil). Ecology of Freshwater Fish 6:123-133.

Alvim, M. C. C. \& Peret, A. C. 2004. Food resource sustaining the fish fauna in a section of the Upper São Francisco River in Três Marias, MG, Brazil. Brazilian Journal of Biology 64(2):195-202.

Bennemann, S. T.; Capra, L. G.; Galves, W. \& Shibatta, O. A. 2006. Dinâmica trófica de Plagioscion squamosissimus (Perciformes, Sciaenidae) em trechos de influência da represa Capivara (rios Paranapanema e Tibagi). Iheringia, Série Zoologia, 96(1):115-119.

Casatti, L. 2003. Family Sciaenidae. In: Reis, R. E.; Kullander, S. O. \& Ferraris JR., C. J. eds. Check list of the freshwater fishes of South and Central America. Porto Alegre, EDIPUCRS. p.599-602.

Durães, R.; Pompeu, P. S. \& Godinho, A. A. L. 2001. Alimentação de quatro espécies de Leporinus (Characiformes, Anostomidae) durante a formação de um reservatório no sudeste do Brasil. Iheringia, Série Zoologia, 90:183-191.

Fugi, R.; Hahn, N. S. \& Agostinho, A. A. 1996. Feeding styles of five species of bottom-feeding fishes of the high Paraná River. Environmental Biology of Fishes 46:297-307.

Fugi, R.; Agostinho, A. A. \& Hahn, N. S. 2001. Trophic morphology of five benthic-feeding fish species of a tropical floodplain. Revista Brasileira de Biologia 61: 27-33.

Galina, A. B. \& Hahn, N. S. 2004. Atividade de forrageamento de Triportheus spp. (Characidae, Triportheinae) utilizada como ferramenta de amostragem da entomofauna, na área do reservatório de Manso, MT. Revista Brasileira de Zoociências 6(1):81-92. 
Hahn, N. S.; Agostinho, A. A. \& Goitein, R. 1997. Feeding ecology of curvina Plagioscion squamosissimus (Hechel, 1840) (Osteichthyes, Perciformes) in the Itaipu Reservoir and Porto Rico floodplain. Acta Limnologica Brasiliensia 9:11-22.

Hahn, N. S.; Pavanelli, C. S. \& Okada, E. K. 2000. Dental development and ontogenetic diet shifts of Roeboides paranensis Pignalberi (Osteichthyes, Characinae) in pools of the upper rio Paraná floodplain (State of Paraná, Brazil). Revista Brasileira de Biologia 60(1):93-99.

Hellawell, J. M. \& Abel, R. 1971. A rapid volumetric method for the analysis of the food of fishes. Journal of Fish Biology 3:29-37.

Hyslop, E. J. 1980. Stomach contents analysis - a review of methods and their applications. Journal of Fish Biology 17:411-429.

Lammens, E. H. R. \& Hoogenboezem, W. 1991. Diets and feeding behaviour. In: Wintfield, I. J. \& Nelson, J. eds. Cyprinids fishes systematics, biology and exploitation. London, Chapman and Hall. p.353-376

Lima-Junior, S. D. \& GoITEN, R. 2003. Ontogenetic diet shifts of a Neotropical catfish, Pimelodus maculatus (Siluriformes, Pimelodidae): An ecomorphological approach. Environmental Biology of Fishes 68:73-79.

Lowe-Mcconnell, R. H. 1987. Ecological studies in tropical fish communities. Cambridge, Cambridge University. 382p.

Luiz, E. A.; Agostinho, A. A.; Gomes, L. C. \& Hahn, N. S. 1998. Ecologia trófica em peixes de dois riachos da bacia do rio Paraná. Revista Brasileira de Biologia 58 (2):273-285.

Mendonç, F. P.; Hahn, N. S. \& Loureiro-Crippa, V. E. 2004. Feeding aspects of Leporinus amblyrhynchus (Characiformes: Anostomidae) in the first stages of formation of a neotropical reservoir, Brazil. Neotropical Ichthyology 2(3):145-150.

Mourão, G. M. \& Torres, G. E. 1984. Espectro alimentar e atividade predatória da corvina, Pachyurus squamipinnis (Pisces, Sciaenidae) no reservatório de Três Marias, rio São Francisco, MG. Anais do Seminário Regional de Ecologia 4: 295-309.

Novakowski, G. C.; Fugi, R. \& Hahn, N. S. 2004. Diet and dental development of three species of Roeboides (Characiformes: Characidae). Neotropical Ichthyology 2(3):157-162.

Ortêncio-Filho, H.; Hahn, N. S.; Fugi, R. \& Russo, M. R. 2001. Aspectos da alimentação de Glanidium ribeiroi (Haseman, 1911) (Teleostei, Auchenipteridae), espécie endêmica do rio Iguaçu, PR. Acta Limnologica Brasiliensia 13(1):85-92.

Prejs, A. \& Prejs, K. 1987. Feeding of tropical freshwater fishes: seasonality in resource availability and resource use. Oecologia 71:397-404.

Russo, M. R.; Ferreira, A. \& Dias, R. M. 2002. Disponibilidade de invertebrados aquáticos para peixes bentófagos de dois riachos da bacia do rio Iguaçu, Estado do Paraná, Brasil. Acta Scientiarum 24:411-417.

Sabino, J. \& Castro, R. M. C. 1990. Alimentação, período de atividade e distribuição espacial dos peixes de um riacho da floresta Atlântica (sudeste do Brasil). Revista Brasileira de Biologia 50(1):23-36.

TAKEDA, A. M. \& FujITA, D. S. 2004. Benthic Invertebrates. In: Thomaz, S. M.; Agostinho, A. A. \& Hahn, N. S. eds. The Upper Paraná River and its Floodplain: physical aspects, ecology and conservation. Leiden, Backhuys. p.191-205.

Yамамото, K. C. 2004. Alimentação de Triportheus angulatus (Spix \& Agassiz, 1829) no lago Camaleão, Manaus, AM, Brasil. Acta Amazônica 34(4):653-659.

Wainwright, P. C. 1988. Morphology and ecology: functional basis of feeding constrains in Caribbean labrid fishes. Ecology 69:635-645.

Windell, J. T. \& Bowen, S. H. 1978. Methods for study of fishes diets based on analysis of stomach contents. In: Bagenal, T. ed. Methods for assessment of fihs production in fresh water. Oxford, Blackwell Scientific. p.219-226.

Zahorcsak, P.; Silvano, R. A. M. \& Sazima, I. 2000. Feeding biology of a guild of benthivorous fishes in a sandy shore on South-Eastern Brazilian Coast. Revista Brasileira de Biologia 60(3):511-518.

Zaret, T. M. \& RAND, A. S. 1971. Competition in tropical stream fishes: support for the competitive exclusion principle. Ecology 52:336-342. 